\title{
Gastric Lavage Guided by Ultrathin Transnasal Esophagogastroduodenoscopy in a Life-threatening Case of Tobacco Extract Poisoning: A Case Report
}

\author{
Masato Miyauchi', Makiko Hayashida², Kimiko Hirata ${ }^{3}$, \\ Kiyotaka Hirata ${ }^{4}$ and Hiroyuki Yokota ${ }^{1}$ \\ ${ }^{1}$ Department of Emergency and Critical Care Medicine, Nippon Medical School \\ ${ }^{2}$ Department of Legal Medicine, Nippon Medical School \\ ${ }^{3}$ Department of Hygiene and Public Health, Nippon Medical School \\ ${ }^{4}$ Division of Pharmacy, Department of Emergency and Critical Care Medicine, Nippon Medical School
}

\begin{abstract}
Nicotine, which is found in tobacco, is one of the most toxic of all known poisons. A 31year-old woman was brought to our emergency department 2 hours after ingesting a usually fatal dose of a tobacco extract. Although gastric lavage was once commonly used to treat poisoning cases of this type, lavage can lead to such complications as aspiration, hypoxia, oropharyngeal and gastric trauma, and electrolyte disturbances. Recent guidelines have suggested less-aggressive gastric-emptying procedures as initial treatments. Currently, there are no absolute indications for gastric lavage use. The present patient had a history of depressive episodes and had attempted suicide by ingesting an extract derived from 20 cigarettes mixed with alcohol. There was no evidence of vomiting or seizures occurring before arrival of the ambulance. Physical examination revealed no signs of sweating, although the patient appeared to be confused, which is often seen with nicotine intoxication. She admitted using 2 kinds of cigarette, each of which typically contain $11.2 \mathrm{mg}$ of nicotine. The patient's nicotine level was suspected to be higher than 40 to $60 \mathrm{mg}$, which is normally fatal. To determine whether gastric lavage was indicated in this case, we performed ultrathin transnasal esophagogastroduodenoscopy, which neither requires sedation nor compromises the airways. Although 2 hours had passed since ingestion, the tobacco extract and food were directly visualized within the stomach. Subsequently, gastric lavage was performed with 2,000 $\mathrm{mL}$ of water administered through a nasogastric tube. Ultrathin esophagogastroduodenoscopy made it possible to directly observe the gastric lavage and to ensure the stomach contents had been completely removed. No changes were noted in the vital signs, and no obstruction of the airways was observed. The patient recovered quickly and was discharged the following day. Ultrathin esophagogastroduodenoscopy helped determine the diagnosis and ensure that gastric lavage had been performed without complications.
\end{abstract}

(J Nippon Med Sch 2013; 80: 307-311)

Key words: nicotine, endoscopy, gastric lavage

Correspondence to Masato Miyauchi, Department of Emergency and Critical Care Medicine, Nippon Medical School, 1-1-5 Sendagi, Bunkyo-ku, Tokyo 113-8603, Japan

E-mail:m-masa@nms.ac.jp

Journal Website (http://www.nms.ac.jp/jnms/) 


\section{Introduction}

Because nicotine is one of the most lethal poisons known to mankind, tobacco overdose can be lifethreatening. Manifestations of severe nicotine poisoning can include dyspnea, cyanosis, and coma. To prevent death in such cases, the nicotine must be immediately removed. In a previously published position statement ${ }^{1}$ on gastric lavage, gastricemptying procedures were examined. Because lavage can lead to complications, such as aspiration, hypoxia, oropharyngeal and gastric trauma, and electrolyte disturbances, this paper suggested that the initial treatment for acutely poisoned patients should be less aggressive. Furthermore, it stated that there are no absolute indications for gastric lavage in poisoned patients.

In the present case, we ultimately decided whether to perform gastric lavage by directly observing the gastric contents with ultrathin transnasal esophagogastroduodenoscopy (EGD). The EGD procedure can be used without sedation and does not pose a risk to the airways. As such, EGD allowed gastric lavage to be performed safely in our patient, who had ingested a usually fatal dose of nicotine.

\section{Case Report}

A 31-year-old woman, a nonsmoker who had a history of depressive episodes with psychotic elements, attempted suicide by ingesting an extract derived from 20 cigarettes mixed with alcohol. In addition to the tobacco extract, she had also ingested a small amount of antipsychotic medications. Ninety minutes after ingestion, she decided to call an ambulance. There was no evidence of vomiting or seizures before the arrival of the ambulance. When the ambulance arrived at the patient's home, she was conscious, although slightly drowsy, and had a heart rate of 114 beats/ minutes and a blood pressure of 120/80 mm Hg.

Upon arrival at our emergency department, blood pressure was $116 / 74 \mathrm{~mm} \mathrm{Hg}$, heart rate was 96 beats/minute, respiratory rate was 16 breaths/ minute, and temperature was $36.6^{\circ} \mathrm{C}$. The oxygen saturation was $100 \%$ on $100 \% \mathrm{O}_{2}$, and the Glasgow Coma Scale score was 15. Physical examination revealed no signs of sweating, although she appeared to be confused, which is often seen with nicotine intoxication. In addition, she began to show bradycardia. When questioned, the patient admitted using both "Kent" and "Lucky Strike" cigarettes, which typically contain $11.2 \mathrm{mg}$ of nicotine per cigarette. Although the dose of the antipsychotic medication that she had ingested with the nicotine extract was not fatal, the serum level of nicotine was suspected to be higher than 40 to $60 \mathrm{mg}$, which is normally fatal ${ }^{2}$.

To confirm the tobacco ingestion, we inserted a nasogastric tube to aspirate the stomach contents. The initial material recovered was a yellow-brown liquid that appeared to contain tobacco. To determine whether gastric lavage was indicated, we performed ultrathin EGD, which can be performed without sedation and without compromising the airways. The patient provided consent before the procedure. Nasal anesthesia was achieved by inserting a catheter coated with $2 \%$ lidocaine and 1 : 5,000 epinephrine gel into the inferior nasal meatus. After 5 minutes, the catheter was removed. We then very slowly inserted the EGD, which was also lubricated with $2 \%$ lidocaine gel, into the inferior nasal meatus (Fig. 1).

Throughout the exam, the patient had no pain, nausea, or vomiting. Nicotine and cotinine concentrations in the serum, urine, and gastric contents were analyzed with gas chromatography/ mass spectrometry in the Department of Legal Medicine, Nippon Medical School. Although 2 hours had passed since ingestion, we were able to directly identify the tobacco extract and food in the stomach (Fig. 2). Because we suspected a fatal dose of nicotine remained within the stomach, we decided to perform gastric lavage. Lavage was performed by passing $2,000 \mathrm{~mL}$ of water through the nasogastric tube until the tobacco extract had been completely cleared from the stomach. Use of EGD made it possible to closely observe the entire lavage procedure. After completing gastric lavage but while still closely observing the stomach, we then 


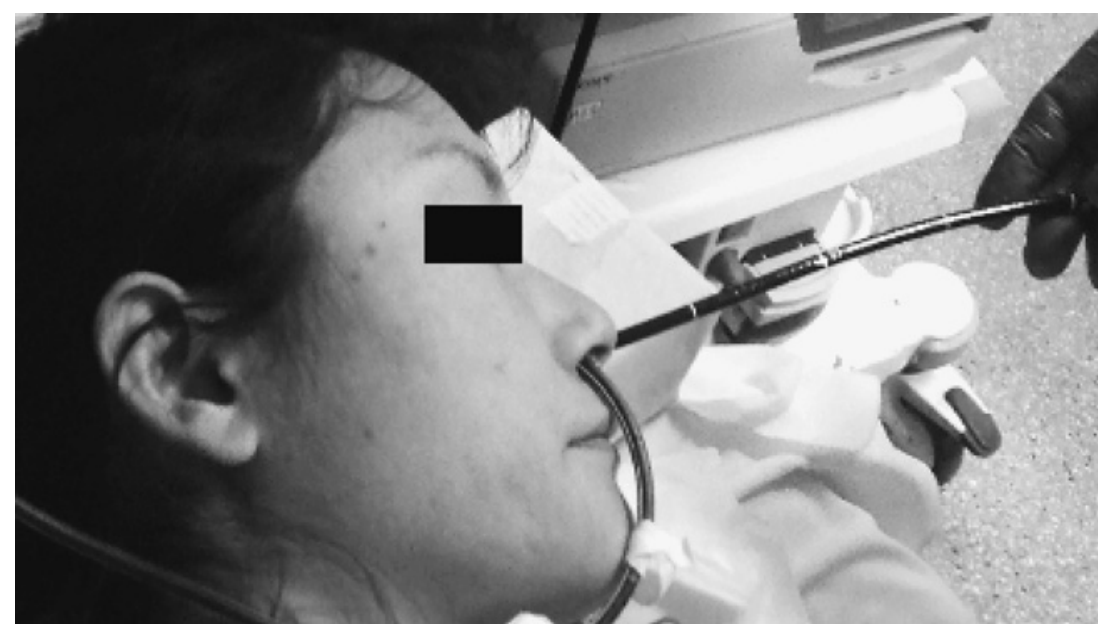

Fig. 1 During the procedure, the ultrathin EGD was inserted through the left nostril while the nasogastric tube was inserted into the stomach via the right nostril.

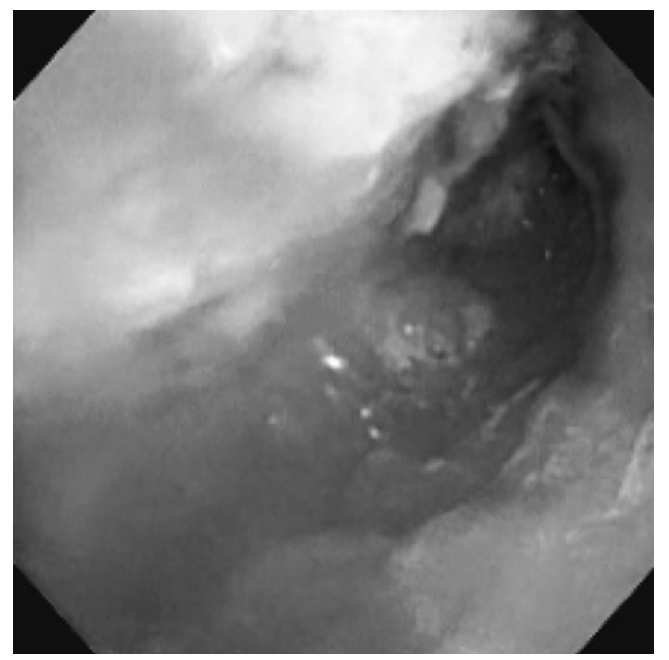

Fig. 2 The endoscopic image shows that both tobacco extract and food remain in the stomach.

administered a single dose of activated charcoal.

During the entire procedure, no changes were noted in vital signs, and there were no complications that posed a danger to the airways. After EGD, the patient was kept under observation in the intensive care unit. Because she remained asymptomatic, she was discharged the following day.

\section{Discussion}

Gastrointestinal decontamination (GID) with gastric lavage was previously commonly performed. However, a position statement and a position paper $^{3}$ concerning gastric lavage have stressed the need for a much less aggressive gastric-emptying procedure during initial evaluation and treatment ${ }^{4}$. Bailey has proposed that patients be evaluated on the basis of a gastrointestinal decontamination triangle ${ }^{5}$. In the first step of this evaluation, whether the ingested substance is likely to have any significant effects is determined. In the second step, whether performing GID is likely to affect the overall outcome is determined. In the third and final step, whether performing GID can harm the patient is considered. With this 3-step evaluation, treatment decisions can be made by assessing the risk of poisoning and the immediate and long-term benefits and risks of the decontamination procedure.

With this evaluation system, we examined the present patient and determined whether gastric lavage was needed. In Japan, the nicotine content of single cigarette ranges from 6.9 to $23.9 \mathrm{mg}$. We suspected that our patient had ingested more than 40 to $60 \mathrm{mg}$ of nicotine, which is normally a fatal dose. In most cases of tobacco ingestion, spontaneous vomiting will usually remove most of the nicotine ${ }^{6}$. The present patient, however, did not vomit. Nicotine reaches a peak plasma concentration 1 hour after oral administration ${ }^{7}$ and has a plasma half-life of 100 to 150 minutes $^{8}$. By the time our patient was admitted to the emergency department, at least 2 hours had passed since she had ingested the tobacco extract. Additionally, although the patient did not 
exhibit dyspnea, cyanosis, or coma, all of which are common in severe nicotine poisoning, the patient began to have bradycardia. Thus, even though a fatal dose of nicotine appeared not to have yet been absorbed, these findings suggested that a fatal dose could soon be absorbed.

Because nicotine is a weak base $(\mathrm{pKa}=8.0)$, it is poorly absorbed from the stomach, and, therefore, we felt that the peak serum concentration had yet to be reached 180 minutes after ingestion. Several factors, including a decreased metabolism due to diet and individual genetic polymorphisms of the CYP2A6 enzyme, can delay peak nicotine serum concentrations $^{9,10}$. As such, we were concerned that the tobacco extract remaining in the stomach might cause vital signs to deteriorate. Consequently, we believed we must try to confirm the contents of the stomach by inserting a nasogastric tube and then, if necessary, aspirating the contents. Unfortunately, even though we confirmed that tobacco extract remained in the stomach, we could not determine the exact quantity.

To further evaluate the gastric contents, we performed ultrathin transnasal EGD. In the past, evaluating the gastric contents has sometimes been recommended when endoscopy is possible and can provide diagnostic information ${ }^{11-14}$. A potential complication of endoscopy is obstruction of the airways. To avoid this complication, we decided to perform ultrathin EGD, which can be performed without sedation and without compromising the airways. Furthermore, because the tobacco extract was liquid, EGD could be used to establish a diagnosis and to monitor subsequent gastric lavage.

Nicotine and cotinine concentrations in the serum, urine, and gastric contents were analyzed with gas chromatography / mass spectrometry in the Department of Legal Medicine, Nippon Medical School. Some of the nicotine in the stomach had metabolized (Table 1) and had caused the cotinine concentration to increase. Fortunately, because the serum nicotine concentration had not reached a critical level, the resulting cotinine levels were not harmful. In addition, although 2 hours had passed since the patient ingested the tobacco extract, no nicotinic intoxication was observed, despite the
Table 1 Concentrations of nicotine and cotinine at admission in serum, urine, and gastric contents

\begin{tabular}{lccc}
\hline & Serum & Urine & $\begin{array}{c}\text { Gastric } \\
\text { contents }\end{array}$ \\
\hline Nicotine $(\mu \mathrm{g} / \mathrm{mL})$ & 0.008 & 0.532 & 14.05 \\
Cotinine $(\mu \mathrm{g} / \mathrm{mL})$ & 0.902 & 0.316 & 1.2 \\
\hline
\end{tabular}

nicotine concentration in the gastric contents being harmful.

The absorption of nicotine can easily be affected by several factors, such as gastric $\mathrm{pH}$ and variations in gastric juice ${ }^{15,16}$. In the present case, we thought that antipsychotic medication, food, and alcohol ingested by the patient in addition to the tobacco extract had likely delayed absorption ${ }^{17}$. However, because the nicotine concentration in the stomach contents remained high, we were concerned that the serum nicotine concentration might continue to increase.

After considering all the factors and evaluating the patient per Bailey's gastrointestinal decontamination triangle, we decided that further intervention was necessary. In addition, because we could still directly observe the stomach, we could effectively and, more importantly, safely perform gastric lavage. It should be noted that even though our findings supported both the indication and the effectiveness of gastric lavage in this case, none of the clinical findings were absolute indications for gastric lavage. However, our results do indicate that ultrathin EGD is safe and can be performed without obstructing the airways. Therefore, we were able to effectively use EGD for both diagnosis and subsequent gastric lavage treatment.

\section{Conclusion}

Ultrathin EGD is an effective diagnostic tool that can also be used without complications during gastric lavage treatment.

Conflict of Interest: The authors declare no conflicts of interest. 


\section{References}

1. Vale JA: Position statement: gastric lavage. American Academy of Clinical Toxicology; European Association of Poisons Centres and Clinical Toxicologists. J Toxicol Clin Toxicol 1997; 35: 711719

2. Garcia-Estrada H, Fischman C: An unusual case of nicotine poisoning. Clin Toxicol 1977; 10: 391-393.

3. Vale JA, Kulig K: American Academy of Clinical Toxicology; European Association of Poisons Centres and Clinical Toxicologists. Position paper: gastric lavage. J Toxicol Clin Toxicol 2004; 42: 933-943.

4. Albertson TE, Owen KP, Sutter ME, et al.: Gastrointestinal decontamination in the acutely poisoned patient. Int J Emerg Med 2011; 4: 65-78.

5. Bailey B: To decontaminate or not to decontaminate? The balance between potential risks and foreseeable benefits. Clin Ped Emerg Med 2008; 9: 17-23.

6. Hoshino $\mathrm{K}$, Yamori N, Arimoto $\mathrm{K}$, et al: Concentration of nicotine and cotinine in infants with tobacco ingestion. Jpn J Pediatr 1996; 100: 1387-1391 (In Japanese with an English abstract).

7. Dempsey D, Tukta P, Jacob P 3rd, et al.: Nicotine metabolite ratio as an index of cytochrome P450 2A6 metabolic activity. Clin Pharmacol Ther 2004; 76: 6472 .

8. Hukkanen J, Jacob P 3rd, Benowitz NL: Metabolism and disposition kinetics of nicotine. Pharmacol Rev 2005; 57: 79-115.

9. O’Loughlin J, Paradis G, Kim W, et al.: Genetically decreased CYP2A6 and the risk of tobacco dependence: a prospective study of novice smokers. Tob Control 2004; 13: 422-428.

10. Zevin S, Benowitz NL: Drug interactions with tobacco smoking. An update. Clin Pharmacokinet 1999; 36: 425-439.

11. Wells CD, Luckritz TC, Rady MY, et al: Bezoar formation requiring endoscopic removal after intentional overdose of extended-release nifedipine. Pharmacotherapy 2006; 26: 1802-1805.

12. Saetta JP, Quinton DN: Residual gastric content after gastric lavage and ipecacuanha-induced emesis in self-poisoned patients: an endoscopic study. J R Soc Med 1991; 84: 35-38.

13. Höjer J, Personne M: Endoscopic removal of slow release clomipramine bezoars in two cases of acute poisoning. Clin Toxicol (Phila) 2008; 46: 317-319.

14. Peeters JW, van der Werf SD: Gastric stenosis after potassium chloride ingestion. Endoscopy 1998; 30: S110.

15. Schneider S, Diederich N, Appenzeller B, et al: Internet suicide guideline: report of a life-threatening poisoning using tobacco extract. J Emerg Med 2010; 38: $610-613$.

16. Allen A, Carroll NJ: Adherent and soluble mucus in the stomach and duodenum. Dig Dis Sci 1985; 30: $55 \mathrm{~S}-62 \mathrm{~S}$.

17. Buckley NA, Dawson AH, Reith DA: Controlled release drugs in overdose. Clinical considerations. Drug Saf 1995; 12: 73-84.

(Received, September 28, 2012)

(Accepted, January 11, 2013) 